Design of the Energy Partitioning Energy Coupling (EPEC) NIF Experiment

J. O. Kane, C. A. Brooksby, C. G. Brown, S. M. Compton, W. H. Dunlop, K. B. Fournier, I. N. Lomov, N. D. Masters, M. J. May, P. B. Mirkarimi, H. A. Scott, D. J. Strozzi, O. Y. Vorobiev, G. B. Zimmerman, R. Guyton, E. J. Huffman, E. A. Smith

May 15, 2013 
This document was prepared as an account of work sponsored by an agency of the United States government. Neither the United States government nor Lawrence Livermore National Security, LLC, nor any of their employees makes any warranty, expressed or implied, or assumes any legal liability or responsibility for the accuracy, completeness, or usefulness of any information, apparatus, product, or process disclosed, or represents that its use would not infringe privately owned rights. Reference herein to any specific commercial product, process, or service by trade name, trademark, manufacturer, or otherwise does not necessarily constitute or imply its endorsement, recommendation, or favoring by the United States government or Lawrence Livermore National Security, LLC. The views and opinions of authors expressed herein do not necessarily state or reflect those of the United States government or Lawrence Livermore National Security, LLC, and shall not be used for advertising or product endorsement purposes.

This work performed under the auspices of the U.S. Department of Energy by Lawrence Livermore National Laboratory under Contract DE-AC52-07NA27344. 


\section{Design of the Energy Partitioning Energy Coupling (EPEC) NIF Experiment}

J. O. Kane, C. A. Brooksby, C. G. Brown, S. M. Compton, W. H. Dunlop, K. B. Fournier, I. N. Lomov, N.D. Masters, M. J. May, P. B. Mirkarimi, H. A. Scott, D. J. Strozzi, O. Y. Vorobiev, G. B. Zimmerman Lawrence Livermore National Laboratory Livermore, CA 94550

R. Guyton, E. J. Huffman, NSTec, Livermore, CA 94550

E. A. Smith, Raytheon KTech, Albuquerque, NM 87123

\section{Summary}

Using scaled experiments performed at the National Ignition Facility (NIF), the Energy Partitioning Energy Coupling (EPEC) experimental platform will provide benchmark data from scaled high-energy-density (HED) environments to validate codes for prompt yield determination following a near-surface explosion. A set of numerical codes has been used to design the experiment and predict the experimental results. Preliminary NIF shots assessing performance of the EPEC energy source (a halfraum) have been carried out. Modeling of the EPEC experiments is discussed, with comparisons to results from the source shots.

\section{Introduction}

Ground shock and air blast overpressure measurements can be used jointly to provide yield estimates for near-surface explosions. The concept of and motivations for the EPEC experiment are discussed in detail in (Fournier 2012); further background is available in (Glasstone).
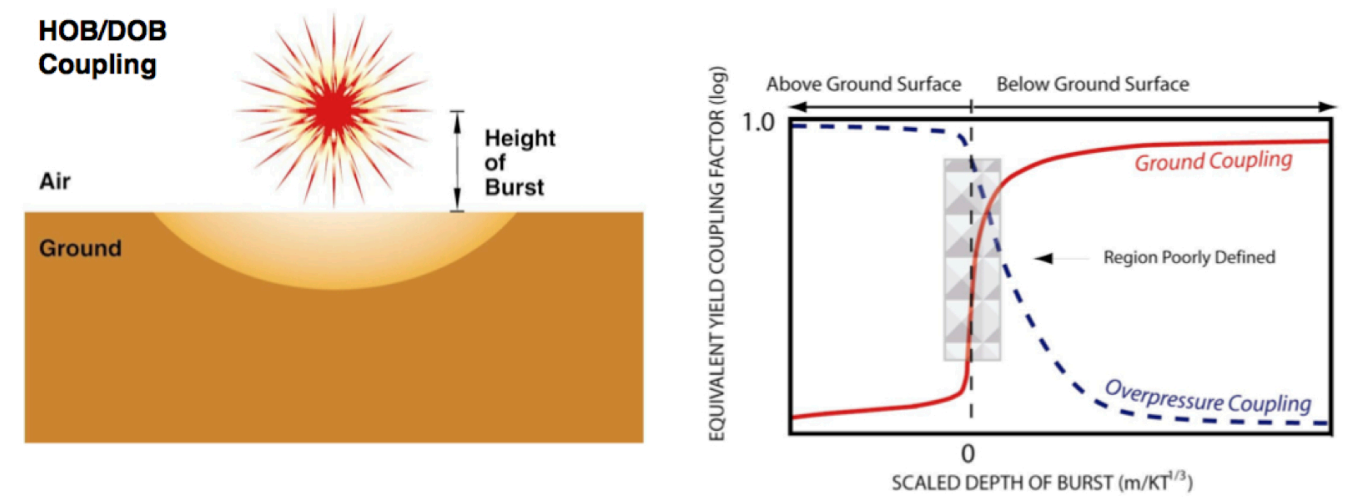

Figure 1. Overpressure coupling and ground coupling. Left: Height of burst or depth of burial determines coupling. Right: the couplings change rapidly in the transition region between above-ground and belowground placement.

The partitioning of blast energy into ground shock and air-blast overpressure close to the surface, both above and below ground, is poorly understood (Figure 1). High 
Explosive (HE) tests cannot be used to probe this poorly understood region, since the relative size of appropriate amounts of HE make HE tests suspect for providing the data. Lasers can create the correct energy density and specific energy in a small enough volume to be useful (Figure 2).

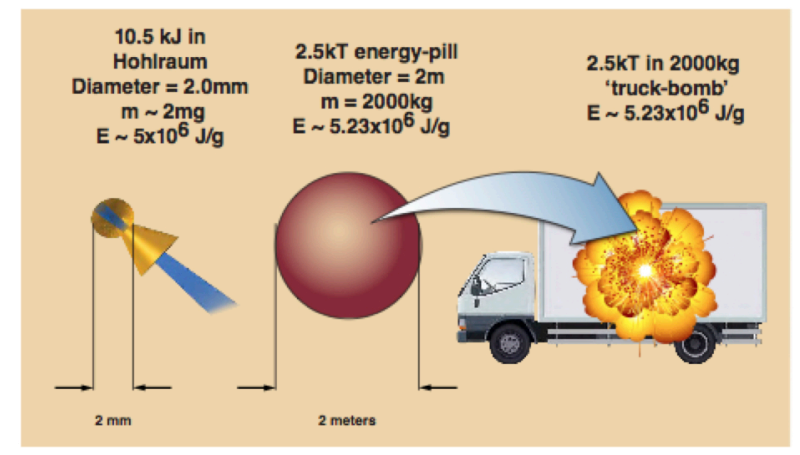

Figure 2. Energy density and specific energy in a NIF target and in the conceptual related scheme.

The Energy Partitioning Energy Coupling (EPEC) High Energy Density (HED) experiments (1) study the partitioning of blast and x-ray energy between seismic and atmospheric responses for known heights of burst and depths of burial, (2) reduce the uncertainty in coupling for near-surface blasts, and (3) may correlate the dynamics of the optical-light emisison from our scaled HED event with a known source energy.

In the EPEC experiments a specialized half-hohlraum ('halfraum') radiation cavity is illuminated with the National Ignition Facility (NIF) laser. In the EPEC 'Energetics' experiments the target is characterized by itself in vacuum. In the EPEC 'Data' experiments, the target is placed inside a polycarbonate cylindrical enclosure containing a mixture of gases representing the atmosphere, and positioned at various distances above and below the surface of a block of BK7 glass representing the ground. The laser light reaches the target through a long, vacuum beam-entry cone. The target disintegration launches blast waves into the atmosphere and the glass. Sensors in the air and the glass record the pressure and stress histories produced by the blast.

The source target behavior is predicted using the LASNEX code (Zimmerman 1975, Harte 1996). The response of the atmosphere and the stresses on the cylinder, cone, sensors and BK7 glass are modeled using the HYDRA code (Marinak 2001). The strain in the cone, end walls and cylinder is modeled using Autodesk (Reference Autodesk) and DYNA3D (Reference DYNA3D). The response of the BK7 substrate is modeling using HYDRA. 


\section{EPEC source target}

The source target in the experiments is a $2 \mathrm{~mm}$ diameter silver $(\mathrm{Ag})$ halfraum having 7-16 $\mu \mathrm{m}$ thick walls and an $800 \mu \mathrm{m}$ diameter laser entrance hole (LEH) where the cone is attached to a $2 \mathrm{~mm}$-long Ag cone with $100 \mu \mathrm{m}$ thick walls (Figure 3). The inside back wall of the target is illuminated through the LEH with 10 $\mathrm{kJ}$ of $0.35 \mu \mathrm{m}$ wavelength laser light using a single quad of NIF. The pulse length is 1 ns and the diameter of the overlapped beam spot at the LEH is $400 \mu \mathrm{m}$ in diameter. Custom continuous phase plates (CPPs) have been made to optimize the beam-spot intensity profile.

The response of the target is measured using a suite of diagnostics, including two broadband Dante spectrometers (Dewald 2004). Figure 4 shows the target from the viewpoints of the two Dante spectrometers. The Dante 1 spectrometer views emission from the LEH; the Dante 1 line of sight is 21.6 degrees from the axis of the target (where 0 degrees would be on-axis looking directly into the target LEH). Dante 2 sees emission from the back outside wall of the halfraum and is 124.1 degrees from the axis.
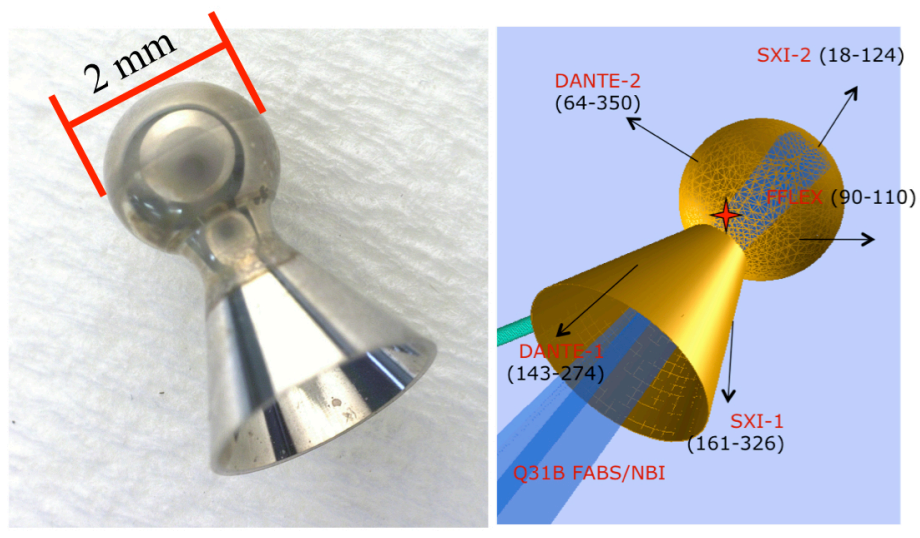

Figure 3. EPEC source target. Left: actual target. Right: directions of drive beams and diagnostic lines of sight.
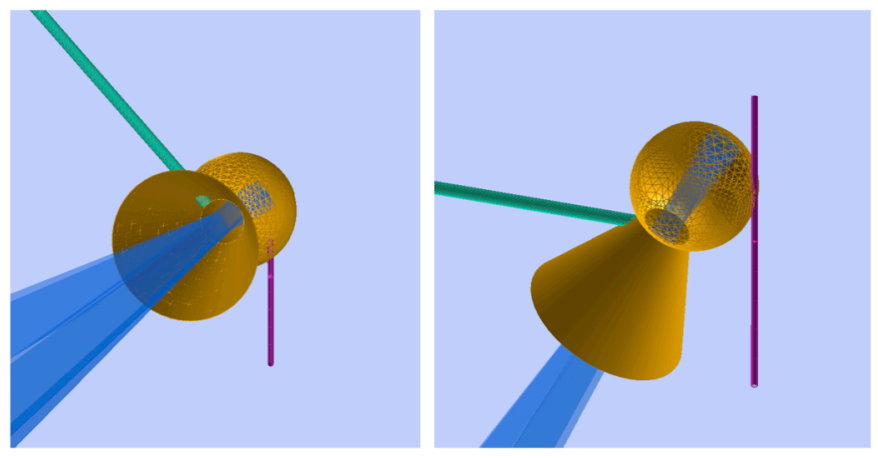

Figure 4. Orientation of EPEC source with respect to target diagnostics. Left: Dante 1 view. Right: Dante 2 view. 


\section{EPEC Cylinder}

In the EPEC Data experiments the target is placed in a $50 \mathrm{~cm}$ long by $12 \mathrm{~cm}$ inner radius cylinder filled with 1.0 atmospheres of a mixture of nitrogen, oxygen and noble gases representing the atmosphere (Figure 5). The target will be driven by four NIF beams delivering $10 \mathrm{~kJ}$ total energy (Figure 6) that enter the polycarbonate cylinder via a beam entry cone; the $2 \mathrm{~mm}$ target cone is mated to the tip of the much larger beam entry cone. The resulting ground shock and air blast dynamic overpressures will be measured by five sensors (provided by Ktech corporation); three of the sensors are embedded in the BK7 glass (see Figure 7). Measurements of the visible light flux that results from deposition in the EPEC atmosphere will be made by two Hammamatsu Photo-Multiplier Tubes (PMTs) in a diagnostics airbox connected to the cylinder.

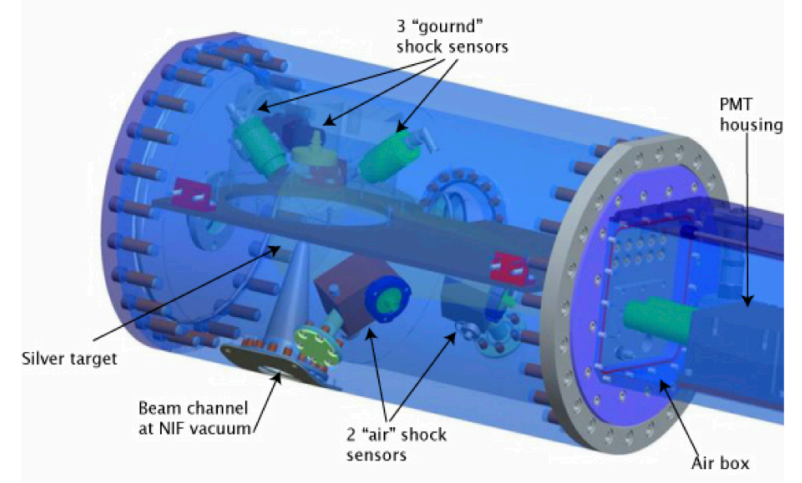

Figure 5. EPEC Data cylinder. The BK7 glass is rendered transparent in this CAD image. The beam entry cone is depicted as entering from the bottom near the right end of the cylinder.

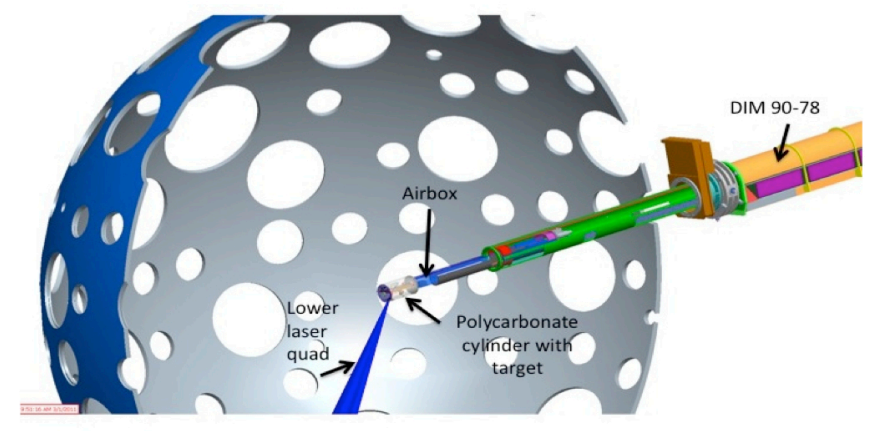

Figure 6. EPEC cylinder and NIF target chamber. The EPEC Data assembly is inserted to target chamber via a Diagnostic Instrument Manipulator (DIM). 


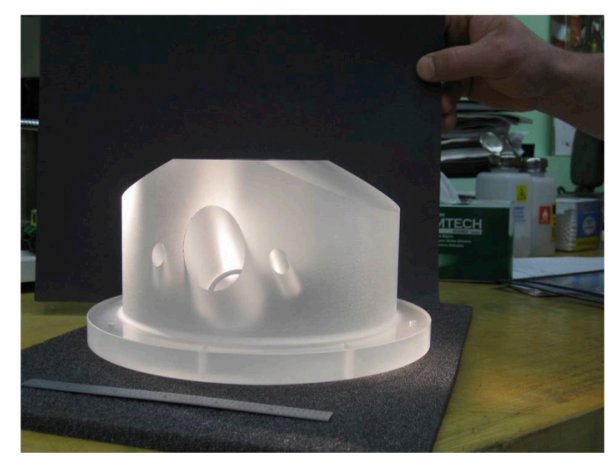

Figure 7. Ground simulant BK7 glass. A precision-ground receptacle for one of the three ground-shock sensors is visible.

\section{Source model}

The target response is modeled using the 2D axisymmetric radiation hydrodynamics LASNEX code, with multigroup Detailed Configuration Accounting (DCA) non-local thermal equilibrium (NLTE) atomic physics (Scott 2011), and a laser ray-trace and absorption package. The interior of the hohlraum is modeled with a 'vacuum' computational mesh containing very low-density hydrogen gas. For extended simulations assessing source spherization and shrapnel transport, an additional air mesh is added outside the halfraum (Figure 8). To model the evolving radiation environment inside the halfraum while limiting inaccuracy caused by numerical diffusion of mass across mesh lines, the models are run with a nearly Lagrangian mesh (co-moving with the material) during the laser pulse and up until the target temperature and emission decrease significantly. For the later-time phase of the extended runs, the mesh is then run Eulerian (held fixed in the laboratory frame) to avoid tangling of the mesh.

DCA is a superconfiguration NLTE model. In NLTE conditions, it may not be true that excited states are described by a Boltzmann distribution, that populations of ionization stages obey the Saha equation, and that opacity and emissivity are related by Kirchhoff's law. DCA is a collisional-radiative model; it describe matter in terms of populations of atomic states, calculates transition rates between levels due to interactions with photons and electrons, and follows the time evolution of populations. Transition rates depend on photon spectrum and the electron distribution. Material properties depend on transition rates and population distributions, and populations can be explicitly time-dependent with non-linear feedback. The photon spectrum depends on material properties through transport. DCA uses a principal quantum number description (superconfigurations), screenedhydrogenic energy levels and rates, and 10-20 levels per ionization stage. DCA includes doubly-excited and autoionizing states, tabulated ionization energies and $\Delta n=0$ transitions (for radiative properties). For the population distributions, forward and reverse rates are related by detailed balance. 

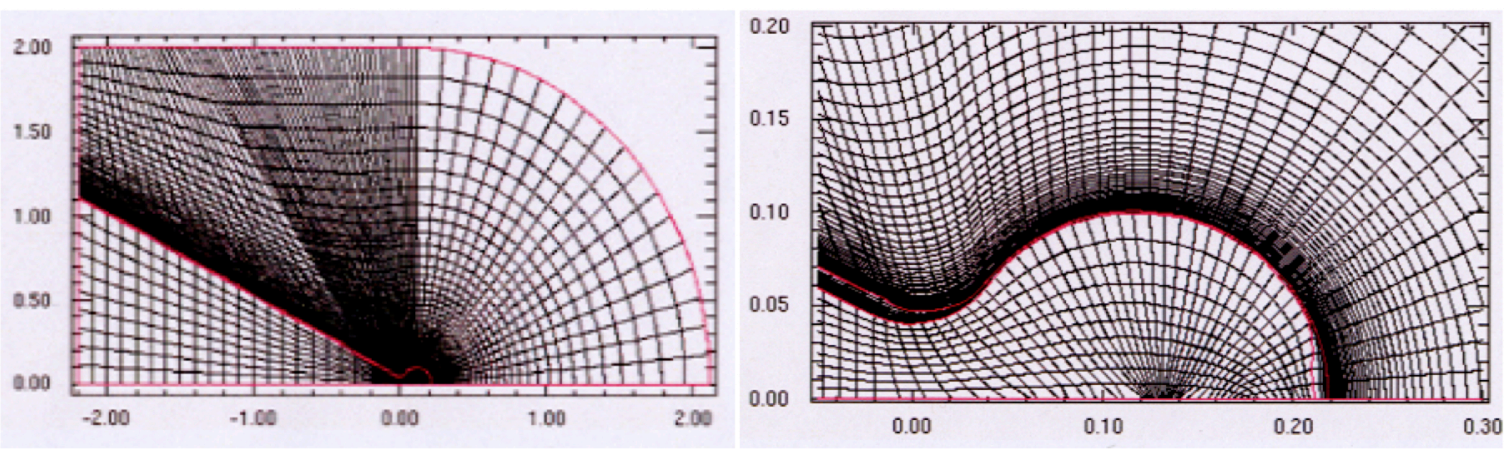

Figure 8. Source model. Left: the full computational mesh with outside air region. Right: close-up view of the halfraum mesh. For simulations of the EPEC energetics shot (halfraum only in vacuum), the outside air mesh is omitted.

\section{Source Evolution}

The evolution of a source halfraum having a $7 \mu \mathrm{m}$ thick wall is shown in Figure 9 by false color plots of radiation temperature $T_{\mathrm{r}}$, electron temperature $T_{\mathrm{e}}$, and electron density $n_{\mathrm{e}}$. During the first 1 ns while the laser pulse is on, Ag plasma is ablated into the halfraum cavity from the inside back wall of the halfraum while a radiative (Marshak) wave propagates supersonically into the Ag wall.
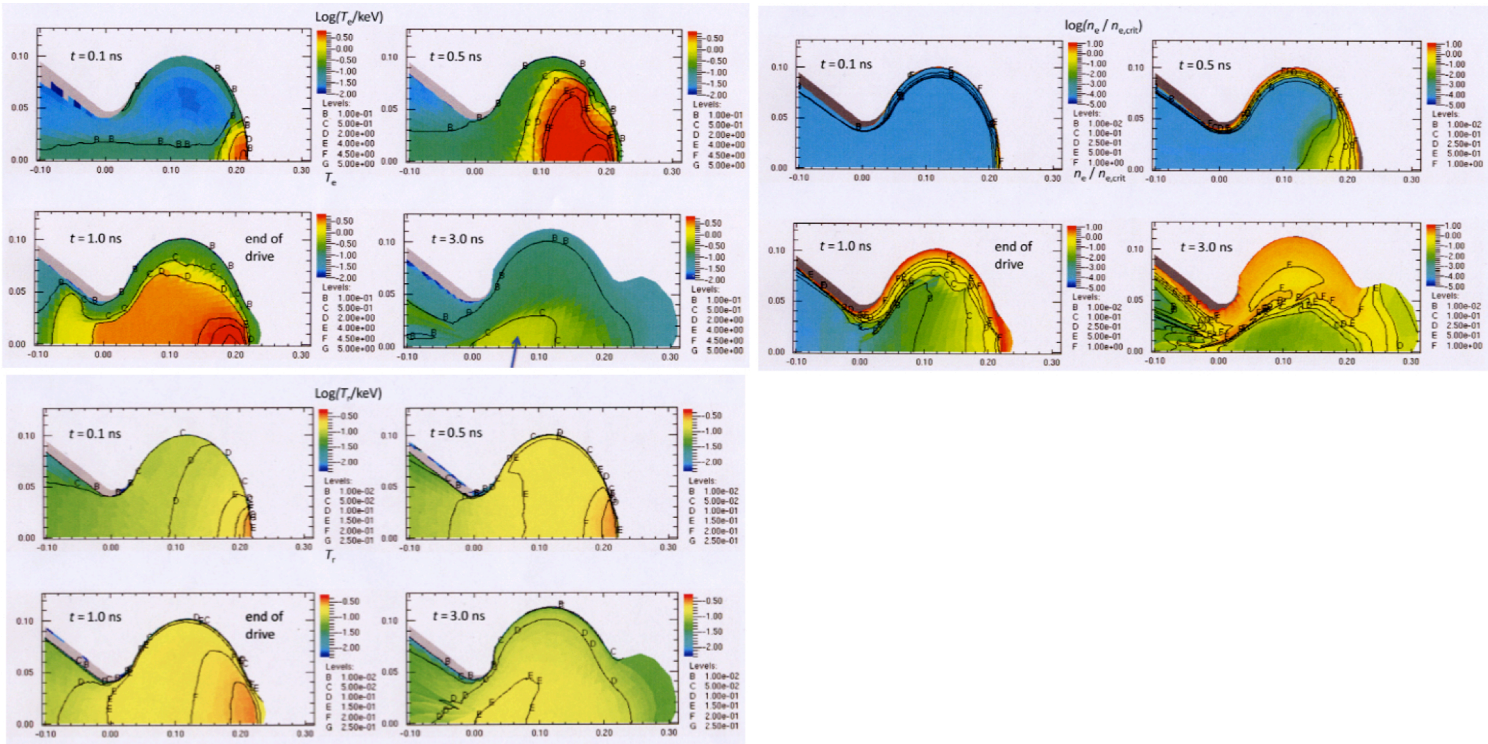

Figure 9. Source evolution: Top left: Electron temperature. Bottom left: Radiation temperature. Right: electron density divided by critical electron density for the $0.35 \mu \mathrm{m}$ laser light.

The hottest radiation temperature $T_{\mathrm{r}}=250 \mathrm{eV}$ occurs near the beam spot. During the pulse, electron temperature reaches a maximum of $T_{\mathrm{e}}>4.5 \mathrm{keV}$ in a broad region of blown-off Ag plasma. In the path traveled by the beams inside the halfraum, electron densities remain below the critical density for resonance absorption, indicating effective absorption of the laser light near the dense wall. By $3.0 \mathrm{~ns}$, a jet of plasma is emerging from the LEH on the halfraum symmetry axis. At this same 
time, the rear wall of the halfraum is burning through, forming a growing bubble of Ag plasma.

\section{DRAT simulated Dante images}

To qualitatively assess the pattern of emission from the halfraum that reaches the Dante spectrometers, the source simulation results are processed with the DRAT utility (Reference DRAT), using simply the parallel-ray approximation, which is a good approximation due to the large (several meter) distance from the target to the spectrometers (Figure 10). DRAT predicts that during the pulse Dante 1 sees a portion of the wall illuminated by the beam spot and some weaker emission shining through the front (source-facing) side of the halfraum. DANTE 2 sees a broader area of emission at lower intensity, with some enhancement near the beam spot.

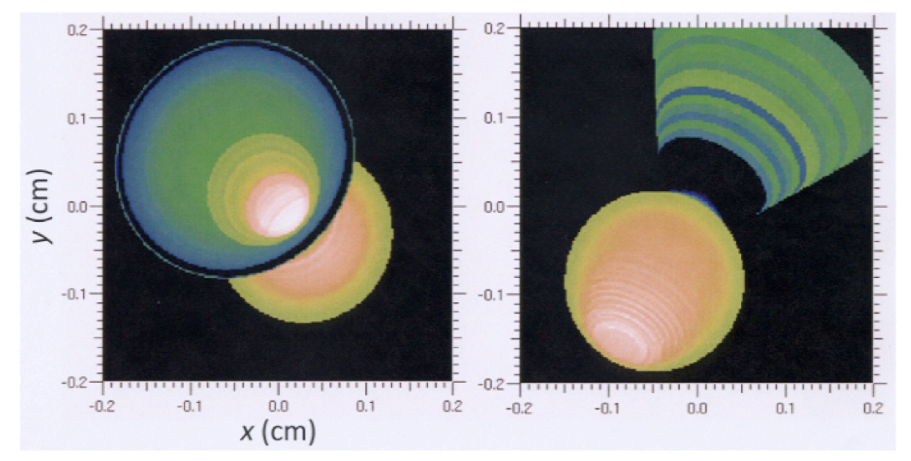

Figure 10. DRAT simulated Dante images for $12 \mu \mathrm{m}$ thick wall.

\section{Dante 1 source spectrum}

Figure 11 shows the predicted spectra seen by the DANTE 1 and DANTE 2 spectrometers for the case of a $12 \mu \mathrm{m}$ thick halfraum wall. Most of the predicted yield occurs below $2 \mathrm{keV}$ photon energy. For a $7 \mu \mathrm{m}$ wall, DANTE 2 sees appreciable emission, while for the thicker $15 \mu \mathrm{m}$ wall (not shown) DANTE 2 sees very low emission due to absorption in the thicker wall.

During the laser pulse the Ag plasma is in NLTE. As a result, the emission below 2 keV consists primarily of an array of lines that are not individually resolved by DCA. The sub-2 keV emission also includes a small thermal (continuum) component corresponding to the $250 \mathrm{eV}$ radiation temperature. 

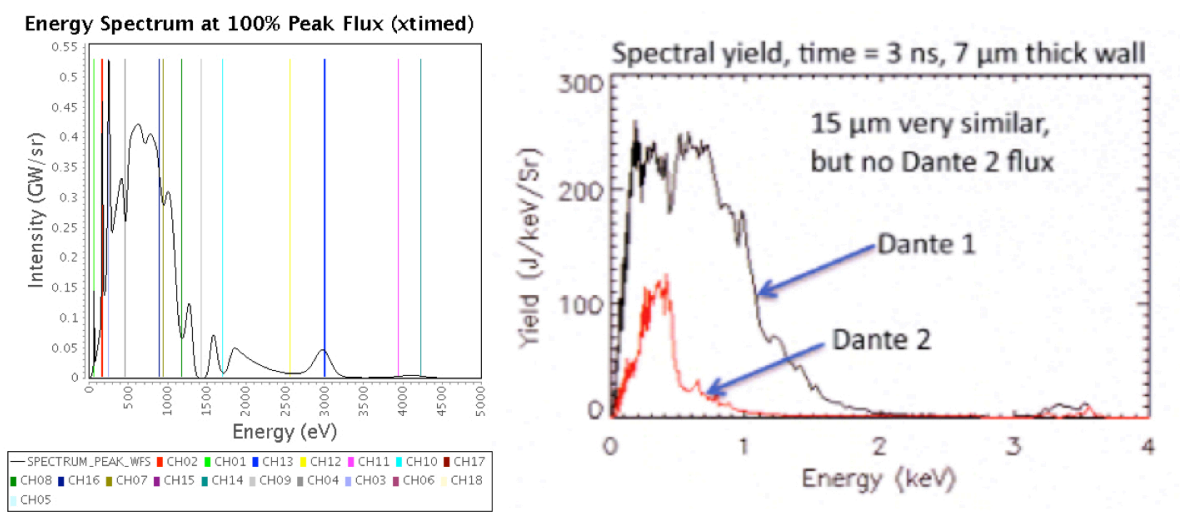

Figure 11. DANTE 1 source spectrum. Left: measured with Dante 1. Right: predictions for a 7 m thick halfraum.

\section{Dante X-ray fluxes}

In Figure 12 the predicted Dante fluxes at photon energies below $5 \mathrm{keV}$ are compared to the DANTE 1 data from EPEC Energetics shots with targets that have $12 \mu \mathrm{m}$ and $15.9 \mu \mathrm{m}$ thick walls. The simulations predict nearly identical results for the two wall thicknesses, because the wall does not burn through during the pulse for either thickness and the wall thickness therefore does not affect the observed emission. The observed DANTE 1 flux is significantly underpredicted. However, the difference between the results for the two targets is similar to the difference between the prediction and the data for the $15.9 \mu \mathrm{m}$-thick wall. At present, we cannot explain why the two targets gave such different results.
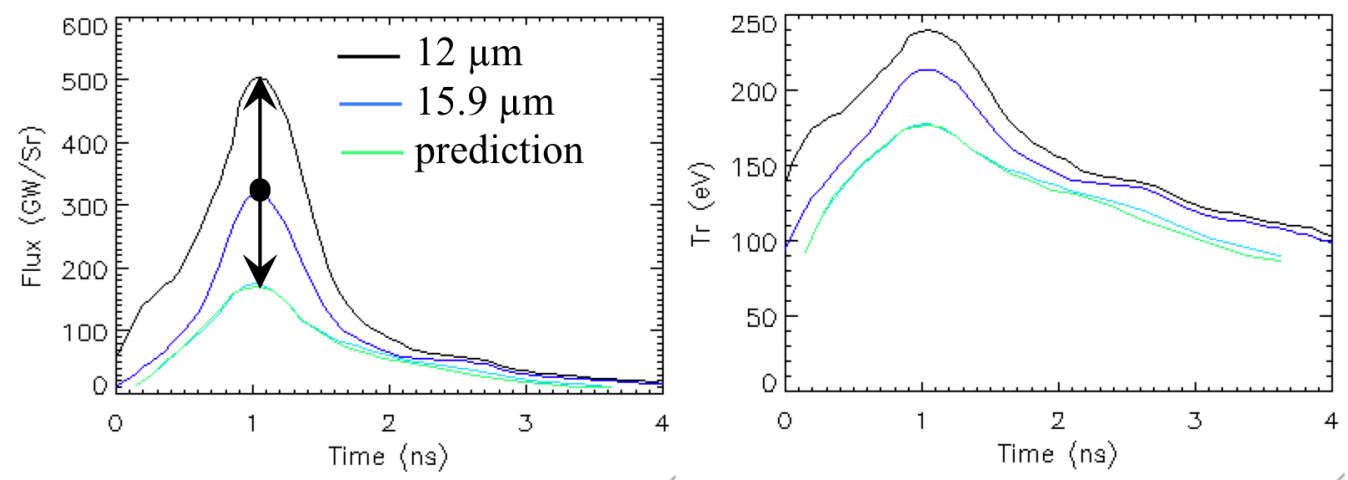

Figure 12. Left: Dante 1 X-ray fluxes. The predictions for the two thicknesses are nearly identical. Right: Corresponding radiation temperatures. 
As shown in Figure 13, the Dante 2 flux is significantly overpredicted. As a check on the simulation, DRAT agrees with the direct flux given by LASNEX. However, the Dante 2 prediction is very sensitive to wall thickness. Furthermore, as seen in Figure 14 , the prediction shows a strong angular dependence, and is sensitive to the version of LASNEX used. With more recent versions of LASNEX, the DANTE 2 yield for a $7 \mu \mathrm{m}$ thick halfraum wall is doubled due to a more sustained later time flux. The differences between the code versions are under continuing investigation.

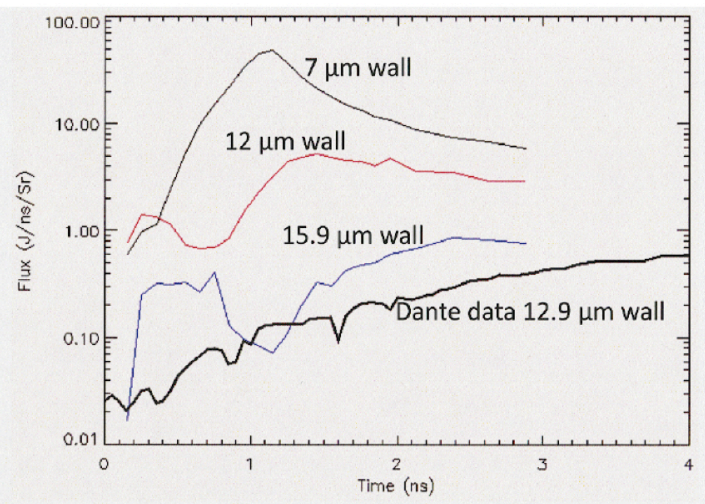

Figure 13. Dante $2 \mathrm{X}$-ray fluxes. Heavy curve: data for $12 \mu \mathrm{m}$ wall. Light curves: predictions.

Also under investigation are the effects of non-uniformity in the drive and possible flaws in the source targets. Upcoming EPEC energetics shots will address both of these issues.

\section{Phase plates and pointing}

Phase plates were not used to smooth out the beam profiles for these source shots. As a result the beam spot contains many hot spots (Figure 15). These hot spots could possibly either be directly visible to DANTE 1 or cause enhanced scattering back out the LEH. In addition, beam mispointing and target misalignment could cause more of the beam spot to be visible to Dante 1 than predicted. Because the LASNEX mesh is 2D axisymmetric it is not capable of representing hot spots. However, LASNEX simulations run with annular beam patterns that have the same area and intensity as nominal spot and a larger maximum radius on the inside wall of the halfraum - potentially allowing more of the bright portion of the beam spot to be visible to DANTE 1, actually predict slightly lower Dante 1 flux. Phase plates for the EPEC experiments have now been manufactured and will be used in the next sequence of EPEC Energetics shots. 

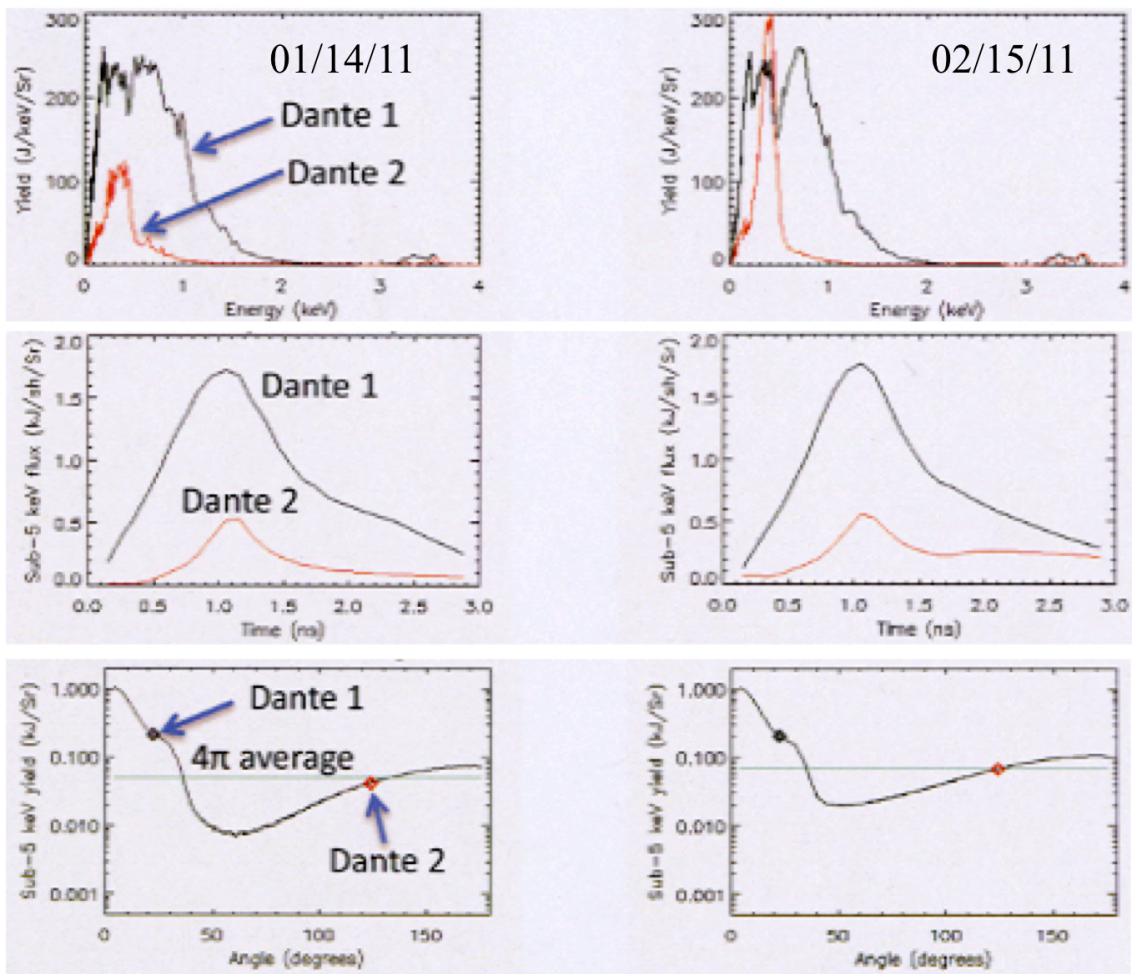

Figure 14. Differences with versions of LASNEX dated one month apart. Top and middle: DANTE 2 yield is doubled with later version due to sustained later time flux. Bottom: the predicted Dante emissions vary strongly with observation angle.
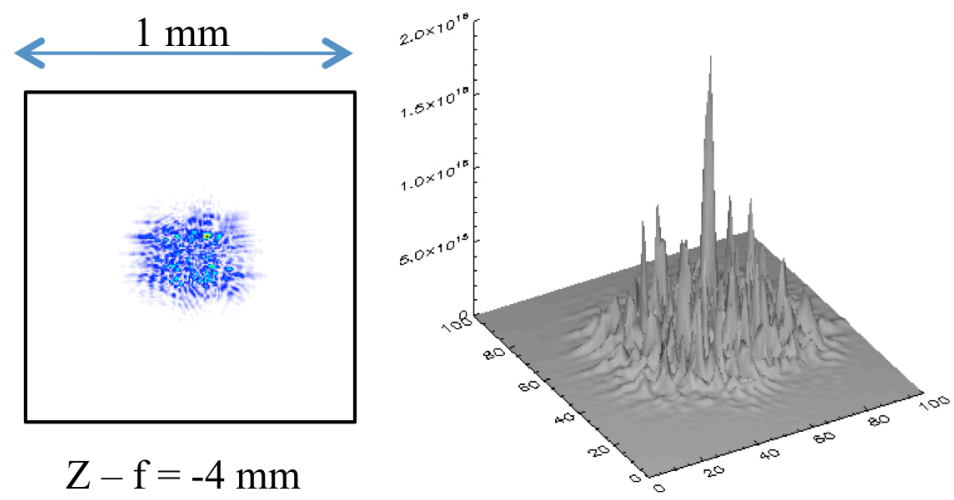

Figure 15. Amplitude of predicted beam hot spots without phase plates.

\section{Al oxide deposits}

Non-destructive and destructive analysis of preliminary source targets supplied by General Atomics suggests the presence of an $\mathrm{Al}$ or $\mathrm{Al}$ oxide layer a few microns thick, apparently with some large chunks also present (Figure 16). These deposits are left over from a stage of the fabrication process in which the aluminum mandrel that supports the very thin halfraum during the delicate machining process is dissolved 
away. These deposits could modify the drive and reduce the Dante 1 and 2 signals, as indicated by results from LASNEX simulations shown in Figure 16. In those simulations, a uniform layer of $\mathrm{Al}$ is added to the inner surface of the halfraum. However, since the simulations without this Al layer already underpredict the Dante 1 data, it appears that simply a uniform Al layer cannot account for the difference between the simulations and data. For upcoming EPEC energetics shots, new targets are being produced using a modified fabrication technique, with a plastic mandrel instead of an aluminum mandrel.
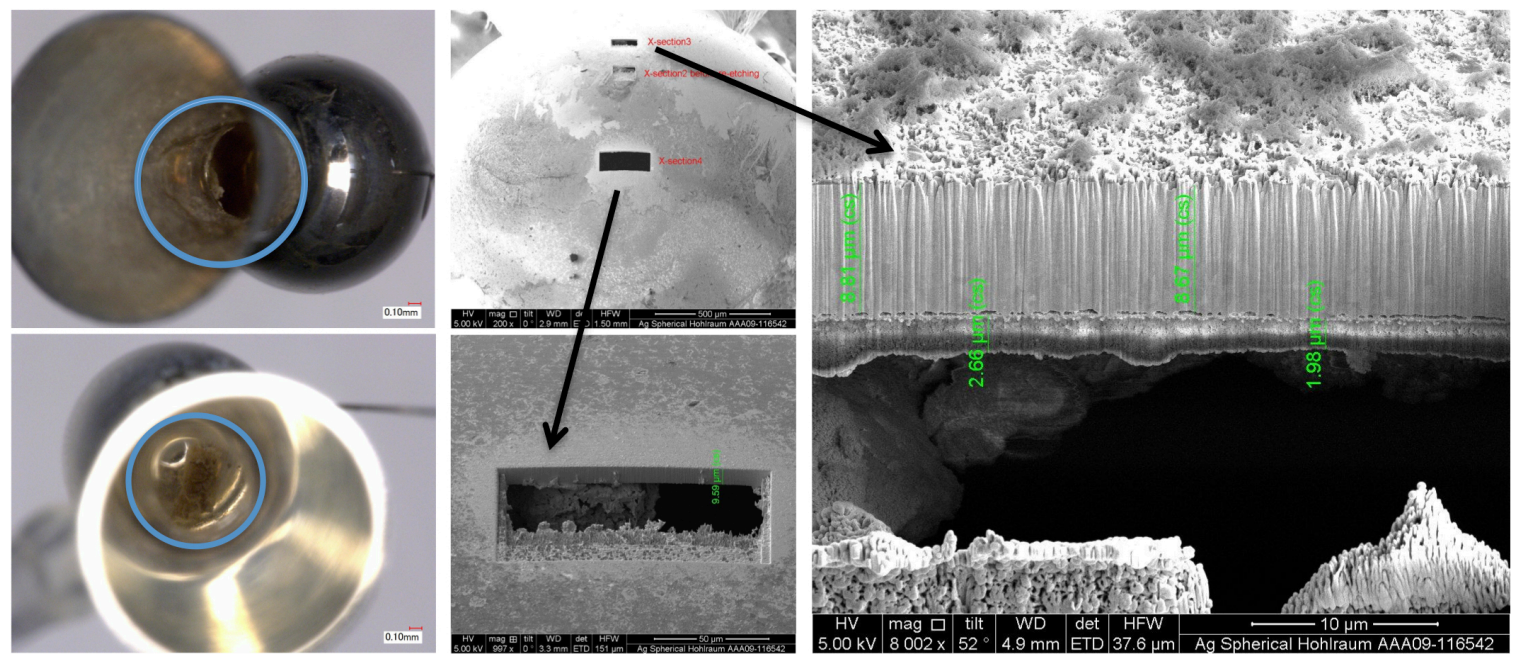

Figure 16. Aluminum oxide deposits in the EPEC Source targets. Left: large chunks near the LEH. Top middle: slits cut into test halfraum. Bottom middle and right: Al layer and Al oxide chunks are evident.
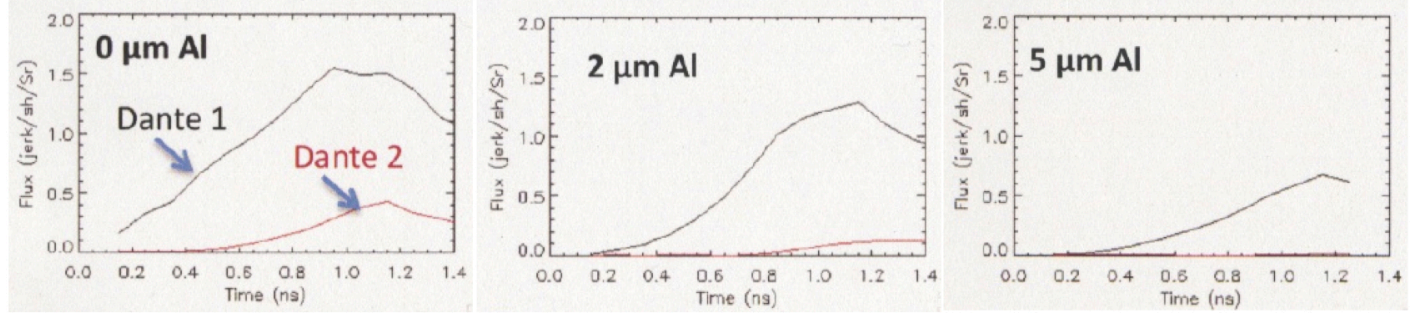

Figure 17. A uniform thickness few- $\mu$ m coating of Al can significantly reduce both the Dante 1 and Dante 2 fluxes.

\section{Analysis of laser plasma interaction}

Laser-plasma interaction (LIP) analysis of the predicted emission backscattered from the halfraum suggest good absorption of laser energy and low risk to the NIF optics; for further details on LIP see (Strozzi 2003). As shown in Figure 18, lowdensity plasma expands from the target wall as time progresses, and plasma stagnates on-axis in LEH. Blue-shifted stimulated Brillouin scattering (SBS) from supersonic expansion off back wall is evident during the pulse, as well as late time stimulated Raman scattering (SRS) and red-shifted SBS from the LEH plasma. 

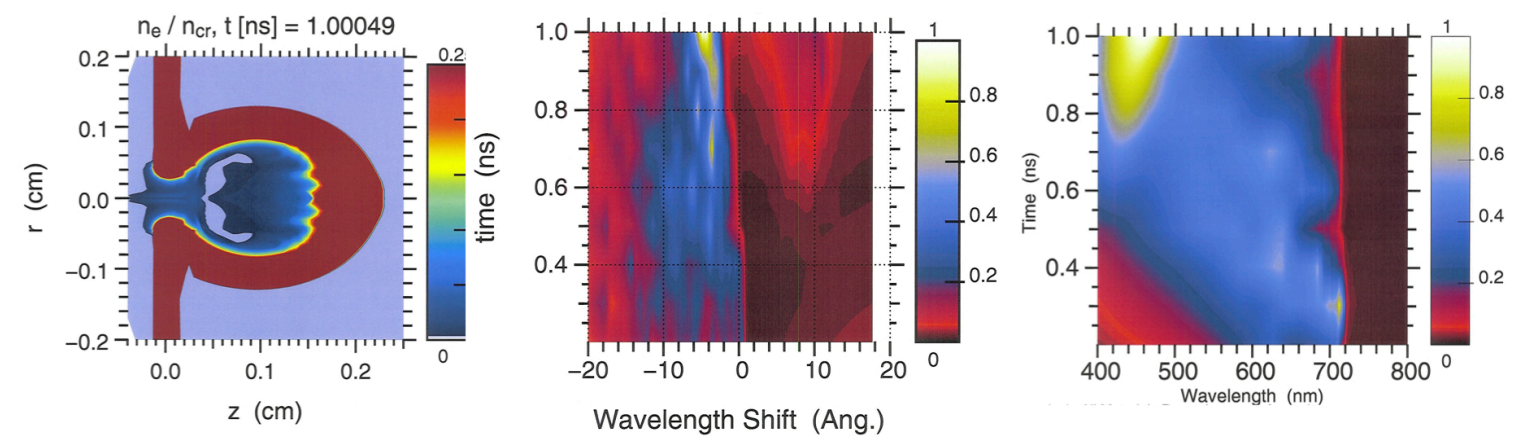

Figure 18. LIP analysis. Left: plasma flow. Middle: SBS: Gain spectra. Right: SRS: Gain spectra.

The analysis suggests that later in the pulse increased backscatter occurs, decreasing the energy available to the EPEC source. With no phase plates, the nonuniformity of the beam spot makes it difficult to predict backscatter levels quantitatively. In general higher beam contrast will enhance backscatter. On the basis of the LIP analysis of EPEC Energetics simulations, the option of using a longer 2 ns pulse was ruled out, because backscatter is worse in that case as the LEH region fills with plasma after the first $1 \mathrm{~ns}$.

Filamentation is predicted near the wall early in time, and later in time at the LEH. This has the effect of increasing the angular spread of the backscatter; by the end of the 2 ns pulse this could result in incident energy missing the LEH. For the $1 \mathrm{~ns}$ pulse, the DEPLETE code (Strozzi 2003) predicts 5\% time-averaged reflectivities. However this does not account for the beam speckle structure; $25 \%$ might be a reasonable estimate. In previous hohlraum experiments (without significant filamentation) at most $10 \%$ of the quad backscatter goes back into a single beamline. This should be an upper bound for these experiments. This gives a fluence of $9.5 \mathrm{~kJ}^{*} 0.25 * 0.10 /(38 \mathrm{~cm})^{2}=0.17 \mathrm{~J} / \mathrm{cm}^{2}$, which is acceptable for the NIF optics. For $2 \mathrm{~ns}$, the corresponding fluence is $10.0 \mathrm{~kJ} * 0.40 * 0.10 /(38 \mathrm{~cm})^{2}=0.28 \mathrm{~J} / \mathrm{cm}^{2}$. Even $100 \%$ backscatter could be tolerated by the laser, because of its poor collimation.

The FABS system measures the reflected laser light (Froula 2009) on NIF by using streaked spectrometers, fast and slow photodiodes, near-field cameras, and time integrated spectrometers to measure independently the characteristics of both the laser light scattered from ion-acoustic waves and from plasma-electron waves in the target plasma for each of the four incident laser beams in our drive quad. The measurement is accurate to $\pm 18 \%$ over a range of backscattered energy from $5 \mathrm{~J}$ to 5 $\mathrm{kJ}$. Based on the measured laser energy reflected from our two targets, the laser-totarget coupling was $93 \%$ of the delivered laser energy. This is excellent coupling, and provides the energy source that will drive blast phenomena in the full EPEC systems to be used in the Data Campaign. The measured loss of $7 \%$ of the delivered energy is in good agreement with the $5 \%$ loss predicted by the LIP analysis. 


\section{Extended LASNEX calculations}

Extended LASNEX calculations run to later times are performed to assess the degree to which the EPEC Data source is effectively point-like, and also address the possibility of shrapnel formation that could impact NIF optics. The extended simulation includes a large air mesh outside the halfraum (Figure 8). The outside mesh contains the mixture of dry air and noble gases used in the EPEC Data experiments. At early time emission from the back wall of the halfraum begins to raise the pressure in the air next to the back wall, creating a bubble of heated gas. As the halfraum burns through and then disintegrates and expands, a larger bubble forms, which clearly begins to spherize by $100 \mathrm{~ns}$ (Figure 19). The timescales for the evolution of the blast wave in the atmosphere in the EPEC Data experiment is from $\mu$ s to ms, suggesting that the source effectively generates a pointlike explosion.

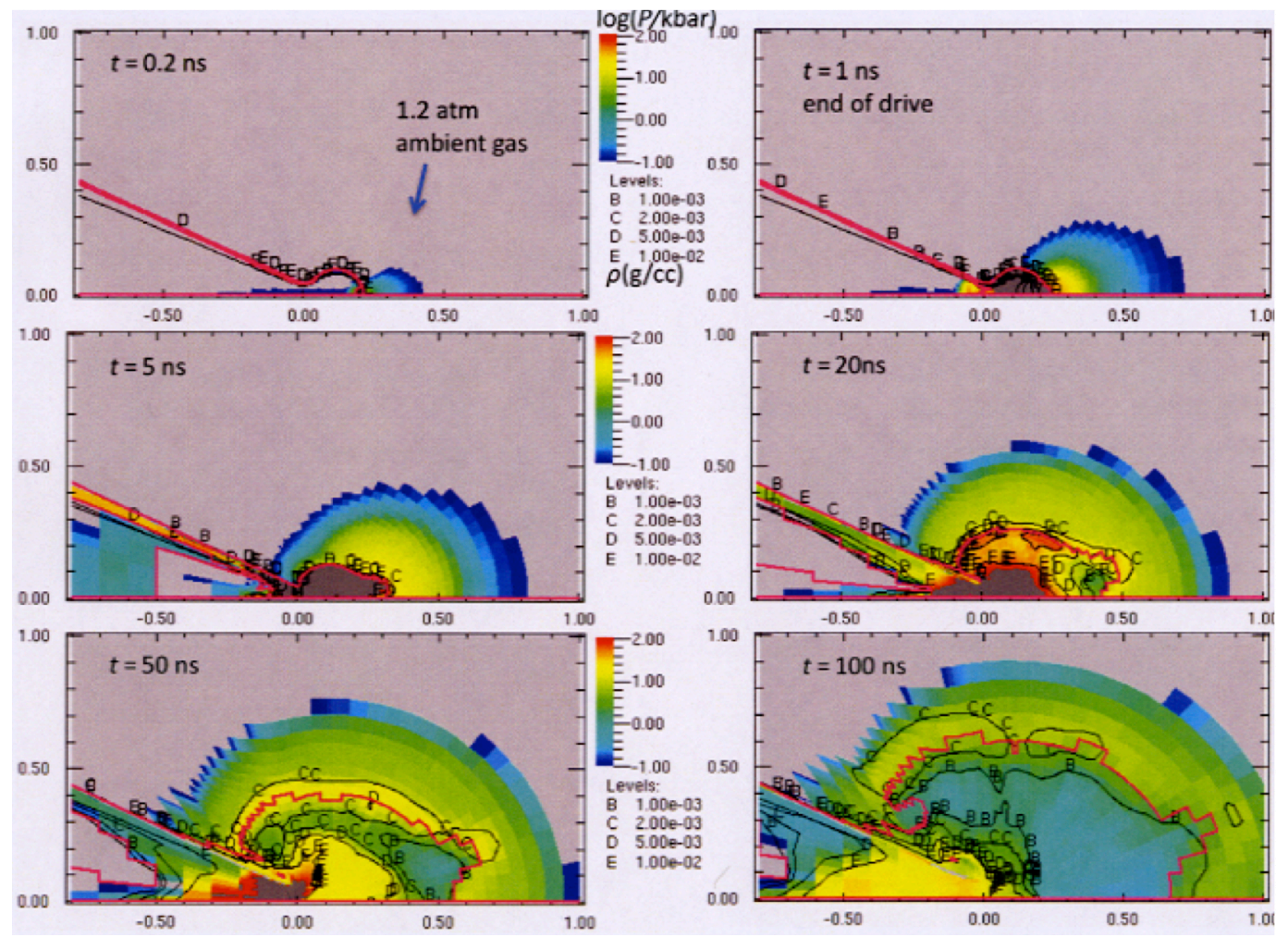

Figure 19. The source spherizes at late times.

The mass of the halfraum is approximately $m=1 \mathrm{mg}$ for the $7 \mu$ thick wall, and $2 \mathrm{mg}$ for the $15 \mu \mathrm{m}$ thick wall. At approximate density $\rho=1.2 \mathrm{e}-3 \mathrm{~g} / \mathrm{cm}^{-3}$, the equivalent mass of the air mixture is contained in a volume of radius $R=\{\mathrm{m} /[(4 / 3) \pi \rho]\}^{1 / 3} \approx 3$ $\mathrm{mm}$, compared to the $1 \mathrm{~mm}$ radius of the halfraum. The energy of the explosion is approximately $\mathrm{E}=5 \mathrm{~kJ}=5 \mathrm{e} 10 \mathrm{erg}$ or greater. The background thermal energy density at room temperature is approximately $e=(3 / 2) n k T=1.5 \times 1 \mathrm{e}-19 \mathrm{~cm}^{-3} \mathrm{x}$ $1.38 \mathrm{e}-16 \mathrm{erg} \mathrm{K}^{-1} \times 300 \mathrm{~K}=6 \mathrm{e} 5 \mathrm{erg} \mathrm{cm}^{-3}$, so that energy $\mathrm{E}$ is contained in a spherical volume of gas of radius $R=(E / e)=1 / 3=40 \mathrm{~cm}$ or greater. Therefore a Sedov-Taylor 
description of the explosion (Zel'dovich 2002) should be informative long after the explosion has formed.

The late time simulations are also used as initial conditions for the shrapnel assessment calculations, as shown in Figure 20. Shrapnel assessment calculations performed with the ALE-AMR code (Anderson 2004) indicate no danger to optics from the target cone. The majority of the cone is moving away from the optics and all portions have radial velocities larger than their axial velocities, which means that target remnants move away from the NIF optics debris shields before they reach the target chamber wall. Furthermore, the total mass moving towards the optics is decreasing.
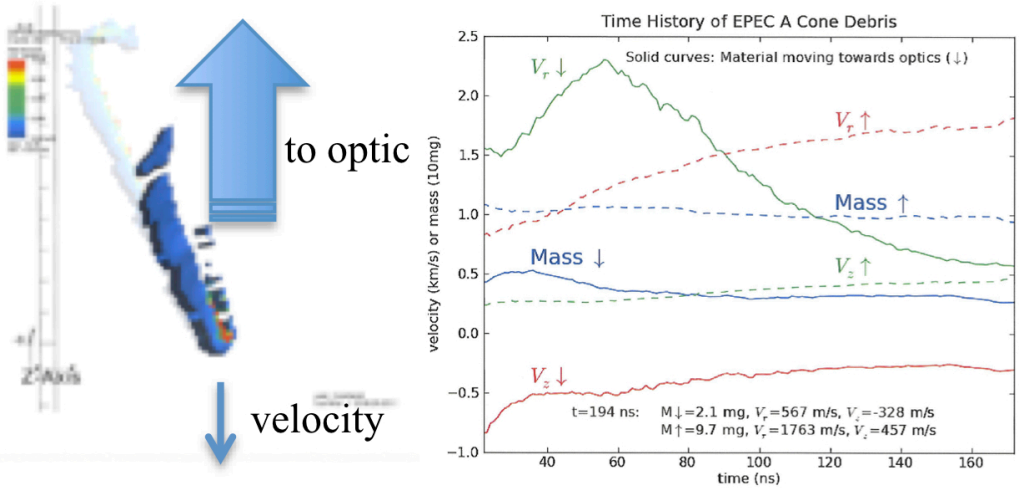

Figure 20. Shrapnel assessment. Left: Density plots of material moving towards the optics at $t=22 \mathrm{~ns}$ and $t=194 \mathrm{~ns}(r>0.5 \mathrm{~g} / \mathrm{cc})$. Right: time history of EPEC cone debris.

\section{Cylinder modeling}

The response of the atmosphere, and the dynamic overpressures on the surfaces of the cylinder, cone, air blast sensors and BK7 glass substrate are modeled using the HYDRA code (Figure 21). The strains in the cone, end walls and cylinder are modeled using Autodesk and spot-checked using DYNA3D. The gas mixture in the 3D HYDRA models is $\mathrm{N}_{2}: 44 \%, \mathrm{O}_{2}: 21 \%$, Ar: $20 \%, \mathrm{Kr}: 10 \%$, Xe: $5 \%$. This mixture is designed to give a scaled man free path for absorption of radiation. Air is about $99 \%$ diatomic; our gas mixture is $65 \%$ diatomic. From ideal gas thermodynamics, the adiabatic exponent is $\gamma=1+2 \mathrm{~N} / \mathrm{F}$, where $\mathrm{N}$ is the total number of particles, and $\mathrm{F}$ is the total number of degrees of freedom, $\mathrm{F}=3 \mathrm{~N}+2 \mathrm{~N}_{\text {diatomic. }}$. Thus $\gamma=1+2 /(3+$ $\left.2 * \mathrm{~N}_{\text {diatomic }} / \mathrm{N}\right)=1+2 /(3+2 * 0.65)=1.47$. 


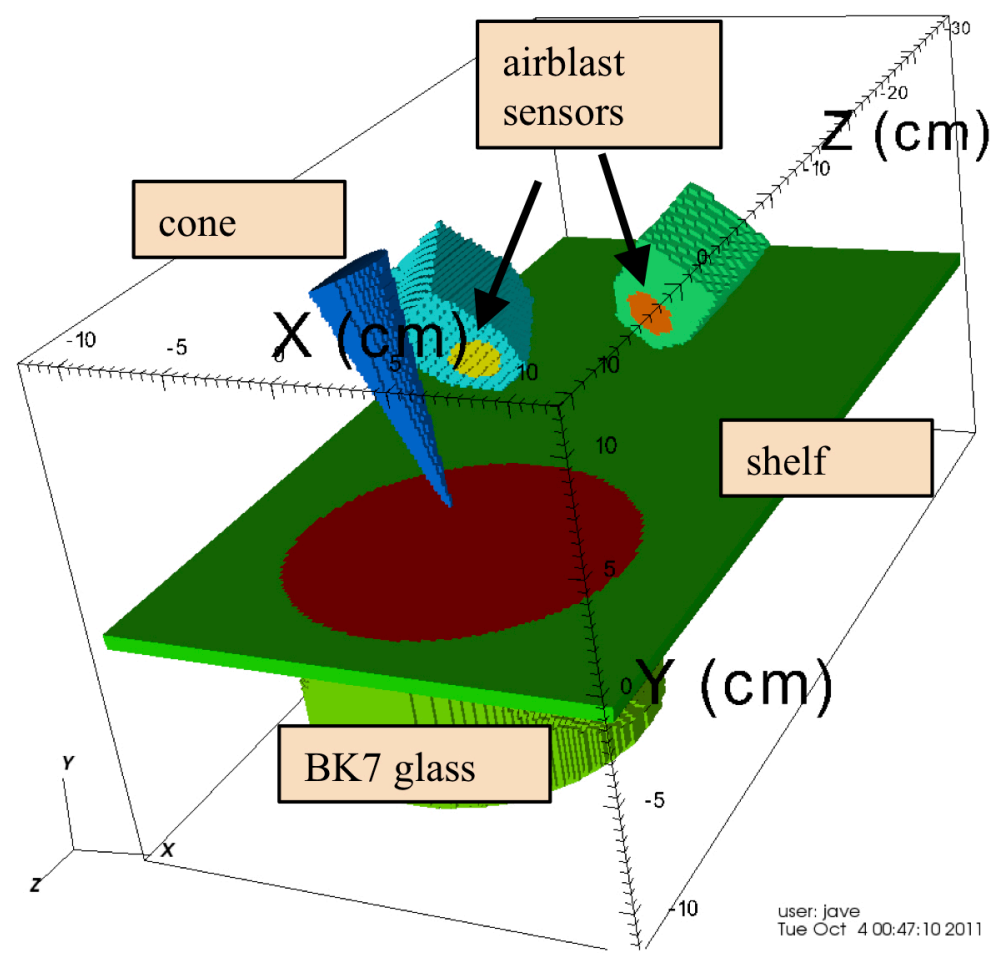

Figure 21. Cylinder modeling. Air, cylinder wall and end plate boundaries are not shown.

To mock up the pressure in the EPEC Data cylinder produced by the blast wave, using 3D HYDRA we model a $12 \mathrm{~cm}$ radius, $50 \mathrm{~cm}$ long cylinder with a $10.5 \mathrm{~kJ}$ thermal 'point' source $1.0 \mathrm{~cm}$ above the 'shelf' ground plane. Boundaries, disk, and cylinder wall are reflecting. The shelf, sensors, and long beam entry cone are modeled as very heavy ideal gas, which effectively acts as an immovable, reflecting solid. We monitor the pressure on the end plate, cone, and cone flange. The model is first checked against the Sedov-Taylor blast wave result for the case of very low background pressure and no cone. The results for the full model are consistent with the expected dynamic overpressure, which includes doubling of thermal pressure on reflection, plus a sharp impulse due to the strong ram pressure. An initially spherical blast wave is distorted by the cone, sensors, shelf, and by reflections from walls (Figure 22). Pressure enhancement occurs where shocks converge, for example where the end plates meet the cylindrical wall. 

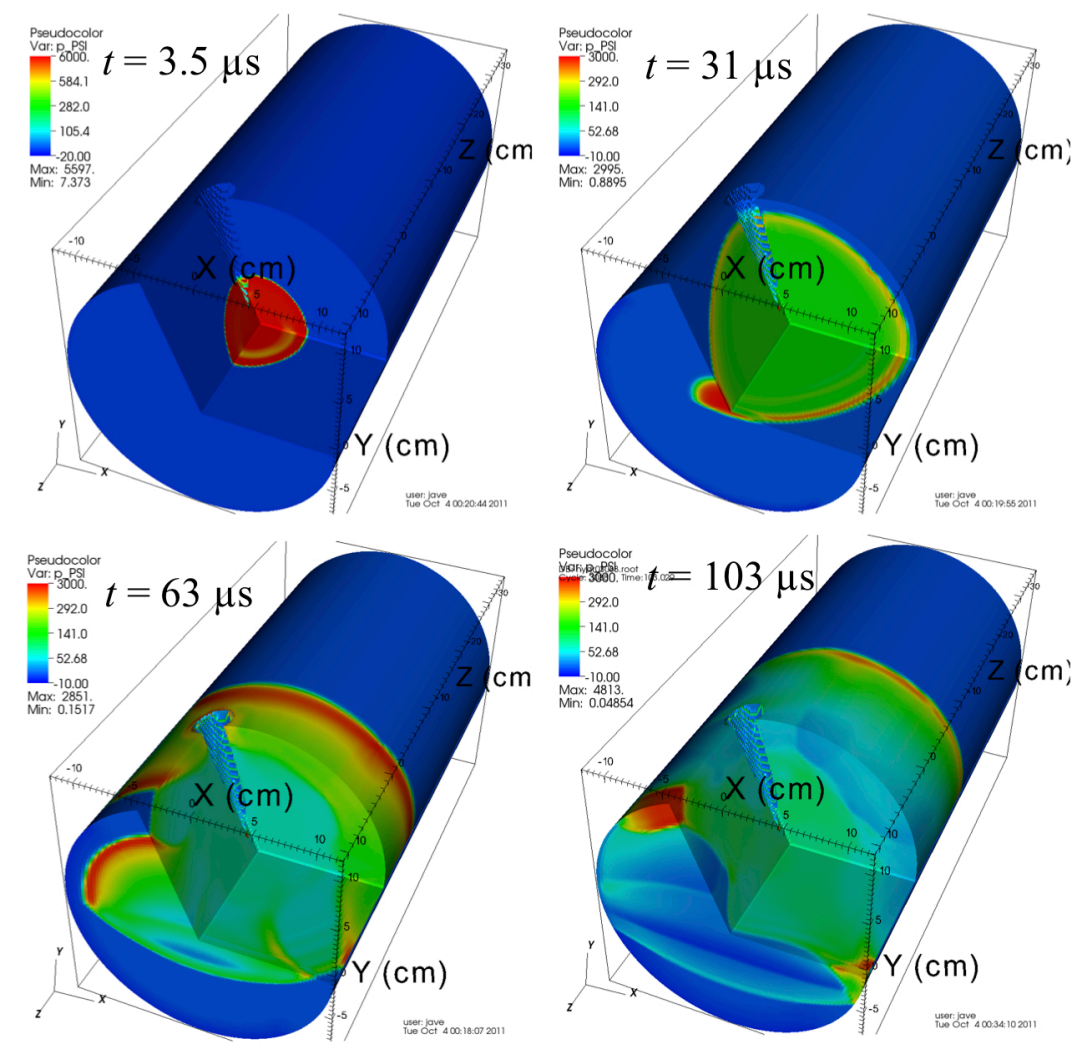

Figure 22. Blast wave interactions inside cylinder.

For the purpose of calibrating the airblast sensors and ground shock pressure sensors, dynamic overpressure profiles are extracted at locations in the atmosphere next to the airblast sensors and the BK7 surface (Figure 23). To assess strain safety factors, dynamic overpressure profiles are extracted in the atmosphere next to the cone, the cone flange connecting the cone to the cylinder, and the aluminum end plates (Figure 24). Using these extracted profiles, Autodesk computes strain safety factors for the cylinder, cone, cone flange, and end plates, with spot checks from DYNA3D (Figure 25)

This combination of HYDRA, Autodesk and DYNA3D modeling has led to a number of refinements to the initial design of the EPEC Data cylinder, including using more bolts to connect the end plates and the cone flange to the cylinder and increasing the thickness of the long EPEC Data beam entry cone connecting the outside chamber vacuum to the source halfraum target. 

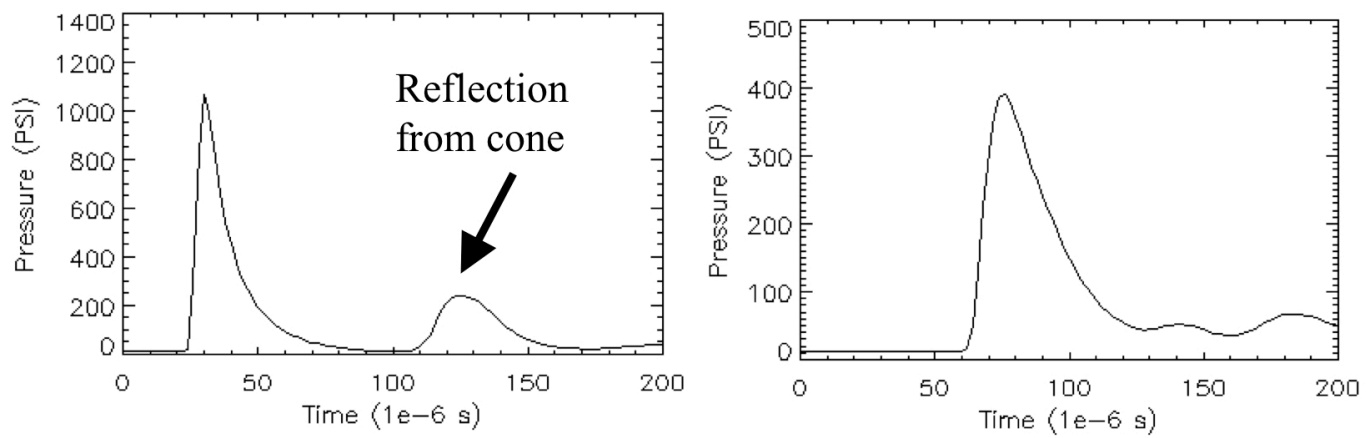

Figure 23. Predicted dynamic overpressure profiles at airblast sensors. Left: Averaged pressure on sensor disc at near sensor (10 cm radius from source). Right Averaged pressure on sensor disc at far sensor (15 cm radius from source).
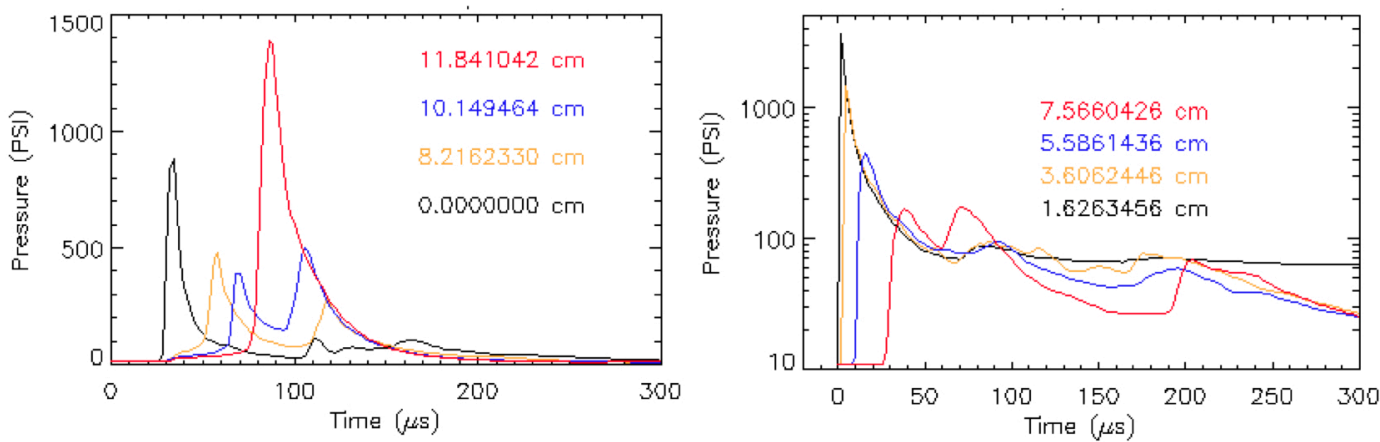

Figure 24. Predicted dynamic overpressure profiles. Left: at various radii on cylinder boundaries. Right: at various heights on the large beam entry cone.
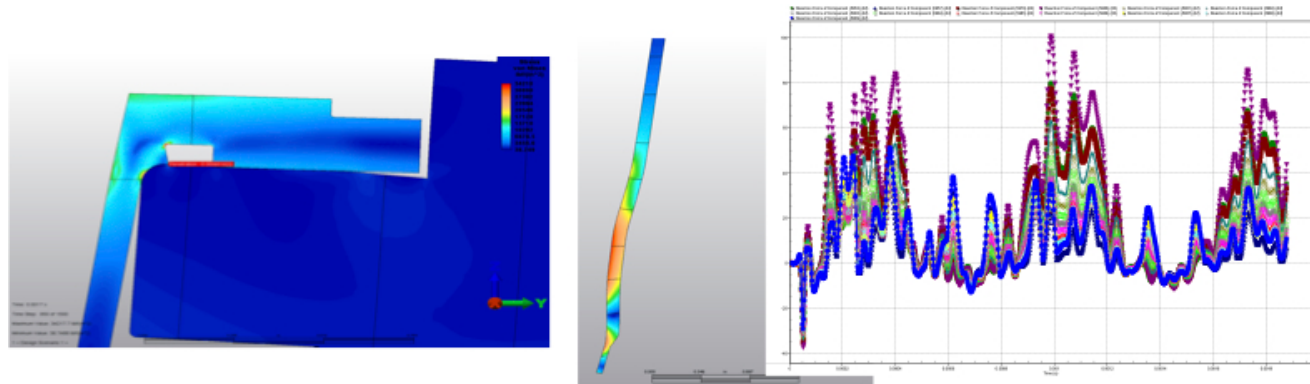

Figure 25. Autodesk stress calculations. Left: Von Mises Stress at 1.7ms \& near max stress of o-ring groove. Middle: VM Stress in cone wall. Right: Screw Z (tensile) forces. Max=100lb. Ave=50lb. 


\section{BK7 response}

Like fused silica, BK7 is amorphous glass and may exhibit a dispersive shock wave, in which case we would expect a ramp wave in the strain sensors embed in the BK7 (Zhuk 1994; Fritz 1978). Since an amorphous glass does not have a crystalline structure, it appears this dispersive behavior would not be due to a phase transition. Besides phase transitions, other discussion of dispersive shock waves in solids in the literature (Zhuk 1994; Fritz 1978) involve collapsing pores, deformation in components of layered materials, and large-amplitude rapid oscillations in the $\mathrm{KdV}$ equation producing an averaged dispersive wave, the application of which seems unclear in this case. The most probable mechanism for a dispersive shock wave appears to be a local densification of the atomic matrix. Currently we are expecting that densification will not occur at the pressures reached in the BK7 in the EPEC data experiment, except possibly near the source, meaning a ramp wave may not be seen at the sensors embedded in the BK7.

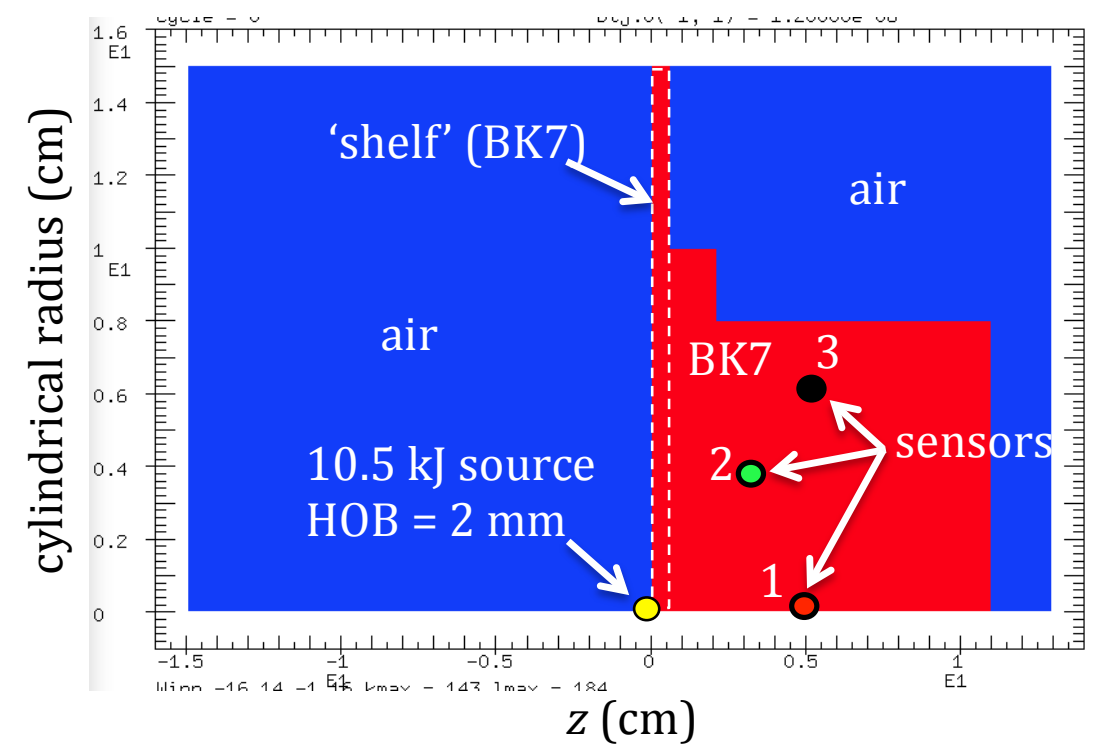

Figure 26. Geometry of HYDRA model of the BK7 response.

As a first step in modeling the BK7 response, we have run a 2D cylindrical HYDRA model containing air and a BK7 substrate (the model is symmetric about the axis of the BK7). A spherical $10.5 \mathrm{~kJ}$ energy source of radius $0.5 \mathrm{~mm}$ positioned in the air 2 $\mathrm{mm}$ or $1 \mathrm{~cm}$ above the surface of the BK7. The geometry of the model is shown in Figure 26 for the case of a $2 \mathrm{~mm}$ height of burst (HOB). For the air EOS we use an ideal gas EOS, with ratio of specific heats 1.47, the same as for the full-cylinder EPEC modeling. For the BK7 model we use a linear Gruneisen equation of state with initial density $2.51 \mathrm{~g} / \mathrm{cm}^{3}$ (no densification), and a strength model with constant shear modulus and 0.2 Mbar yield strength, which in this case is effectively infinite, generating an elastic strength model. The shelf included in the full-cylinder model is represented here by simply extending the BK7; this is intended only to prevent the air blast from wrapping around the BK7 block. Zones were $125 \mu \mathrm{m}$ in size in the 
BK7. The computational mesh was Eulerian (fixed grid) in the air to avoid mesh tangling, and Lagrangian in the BK7 since the displacements are very small there. This model does not capture the impedance mismatch between the BK7 and the sensor material. The mismatch is probably significant for the PVDF sensors (BK7 density is $2.51 \mathrm{~g} / \mathrm{cm}^{3}$; PVDF density is $\sim 1.77 \mathrm{~g} / \mathrm{cm}^{3}$.)

Figure 26 shows the locations of the sensors in the BK7. Sensor 1 is at distance 50 $\mathrm{mm}$ from the point that is on the surface of the BK7 and on the axis ( 0 degrees from the axis). Sensor 2 is located at $50 \mathrm{~mm}$ and 50 degrees; Sensor 3 is at $80 \mathrm{~mm}$ and 50 degrees. Figure 27 shows the normal stress versus time at the sensor locations for the $2 \mathrm{~mm}$ HOB case. Figure 28 is a pseudcolor plot of thermal pressure in the air and BK7 at time $8 \mu$ s after the initiation of the blast. Three waves are visible in the figure: blast waves are propagating directly into the air and BK7 from the vicinity of the source, while ahead of the blast wave in the BK7 a third wave is visible. That third wave is due to the blast initially propagating faster in the air than in the BK7, and then being transmitted from the air into the BK7. The arrival of this early third wave at Sensors 2 and 3 is evident in the top panel of Figure 27. For the 'main' waves at all the sensors, an elastic pullback is evident in Figure 26; the pullback is also visible as the blue-colored wave (indicating negative stress) in Figure 28. For the $1 \mathrm{~cm} \mathrm{HOB}$, the late-arriving wave (transmitted directly in the BK7) catches up to the early-arriving wave at Sensors 2 and 3; this effect along with the lower stress at this larger HOB mitigate the pullback wave at those sensors.

In the experimental measurement, the sensors in the BK7 will not measure arbitrarily sharp profiles; $10 \mathrm{~ns}$ is a realistic floor on the rise time the sensor will see, for two reasons: (1) the sensor has a protective buffer layer several mil thick, which has to ring up to the driving pressure, and (2) due to geometric effects - the nonplanarity and likely irregularity of the shock, the shock arrival time will vary across the surface of the sensor. Figure 29 shows that the early-arriving wave is non-normal at Sensor 2; the sensor surface will be aligned normal to the ray from the point on the BK7 surface on the axis to the center of the sensor pad. 

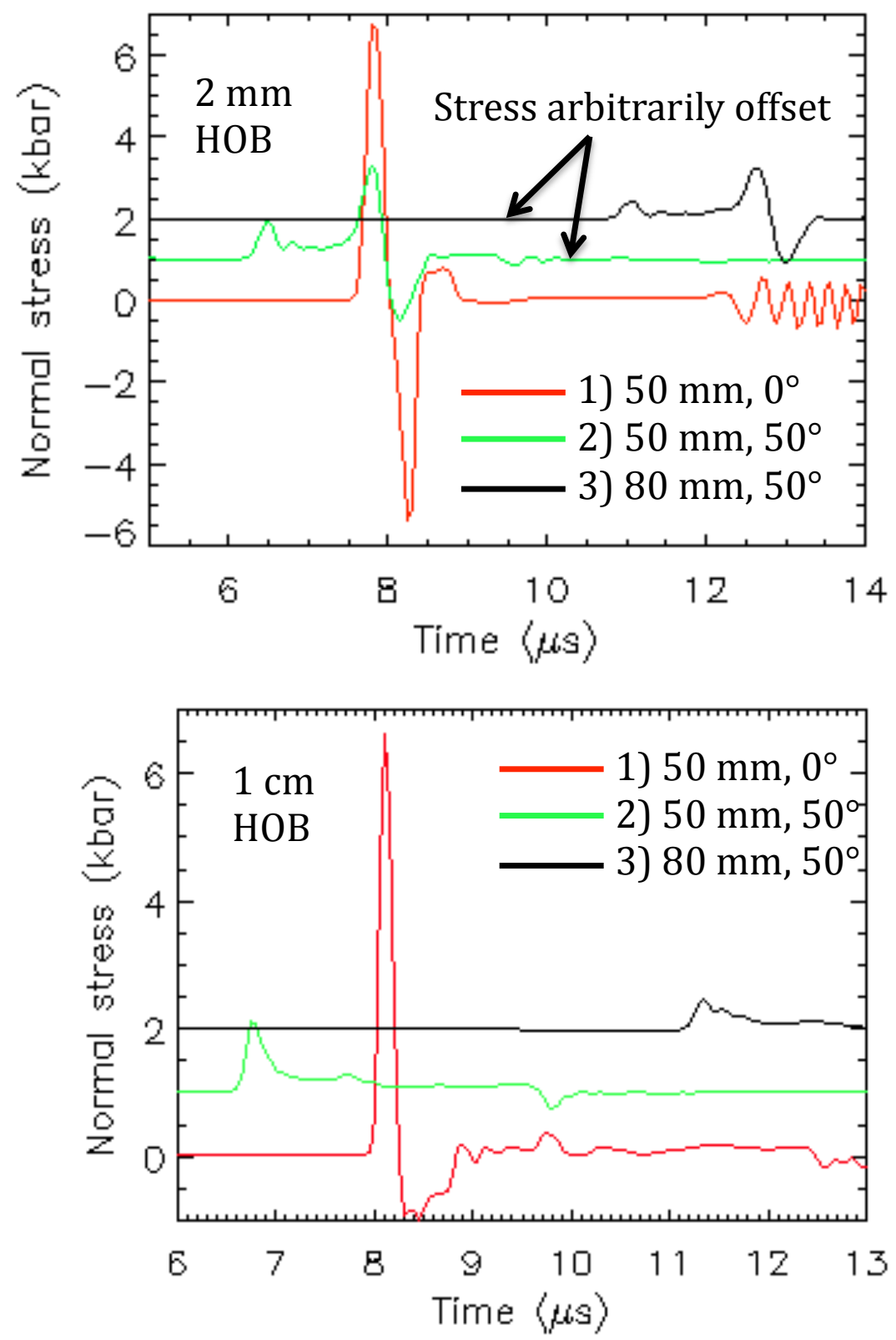

Figure 27. Normal stress vs. time at sensor locations for Top: $2 \mathrm{~mm}$ НОВ case. Bottom: $1 \mathrm{~cm}$ HOB case. For clarity the stresses are plotted with arbitrarily offsets; the flat initial portions correspond to zero stress. 


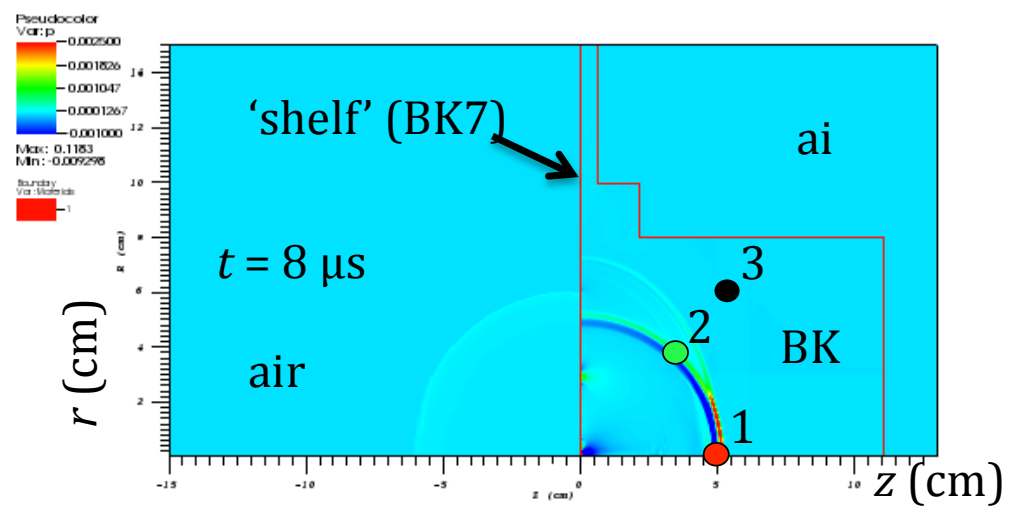

Figure 28. Pseudcolor plots of thermal pressure in the air and BK7 at time $8 \mu s$.
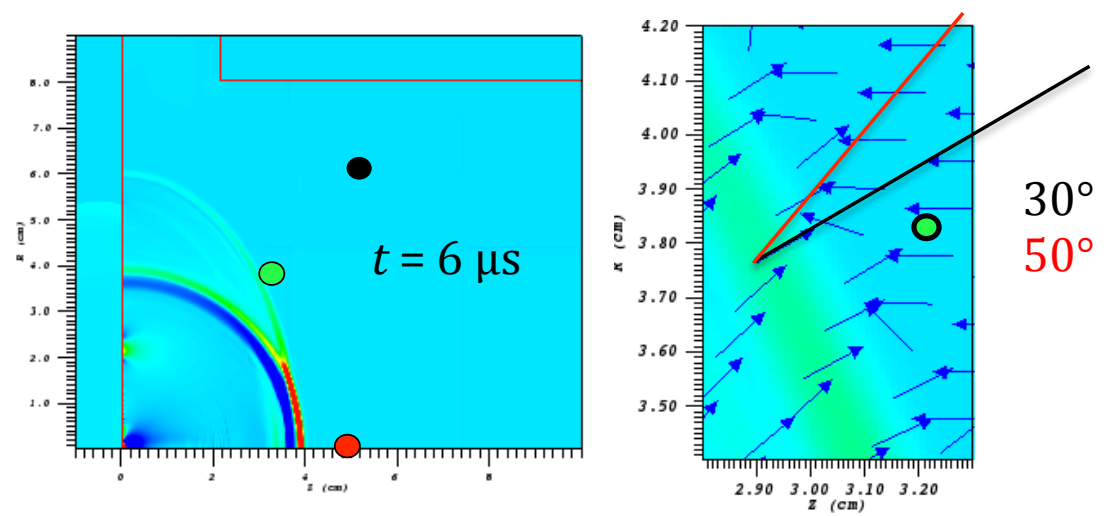

Figure 29. Non-normal arrival of shock front at Sensor 2. The close-up in the right panel shows the normal direction $\left(50^{\circ}\right)$ and the direction $\left(30^{\circ}\right)$ of the velocity vectors whose directions are shown by the arrows.

\section{Integrated modeling}

Integrated modeling that includes the atmosphere as well as realistic EOS and strength models for the solids is being developed. A significant challenge in this modeling is meshing atmosphere around the solid objects, especially around the beam entry cone, in a manner that captures the few- $\mu$ m small displacements over most of the solid surfaces while also allowing the atmosphere to move past those surfaces.

\section{Further EPEC shots}

Further EPEC Energetics shots will be performed in the near future at NIF. Phase plates will be used for these shots, and targets machined on plastic mandrels will be used. The goal of these shots is to generate a more complete and reproducible characterization of the EPEC source, allowing a better assessment of the source modeling. 


\section{Acknowledgements}

*This work performed under the auspices of the U.S. Department of Energy by Lawrence Livermore National Laboratory under Contract DE-AC52-07NA27344.

\section{References}

[Fournier 2012] K. B. Fournier, C. G. Brown Jr., M. J. May, W. H. Dunlop, S. M. Compton, J. O. Kane, P. B. Mirkarimi, "Energy Partitioning, Energy Coupling (Epec) Experiments at the National Ignition Facility", Journal of Radiation Effects, Research and Engineering 30, 119-136 (2012).

[Glasstone] The Effects of Nuclear Weapons (Third Edition), compiled by S. Glasstone and P. Dolan, 1977, US Department of Defense and US Department of Energy.

[Scott 2011] H. Scott and S. Hansen, High Energy Denisty Physics 6 (2010) 39-47; M.D. Rosen et al., "The role of a detailed configuration accounting (DCA) atomic physics package in explaining the energy balance in ignition-scale hohlraums", High Energy Denisty Physics 7, 180 (2011).

[Zel'dovich 2002] Zel'dovich, Ya. B. and Raizer, Yu. P. Physics of Shock Waves and High-Temperature Hydrodynamic Phenomena. Mineola, NY: Dover Publications, 2002

[Strozzi 2003] D. J. Strozzi et al., Physics Of Plasmas 15, 102703 (2008); W. L. Kruer, The Physics of Laser Plasma Interactions (Westview, Boulder, CO, 2003).

[Marinak 2001] Marinak, M. M., Kerbel, G. D., Gentile, N. A., Jones, O., Munro, D., Pollaine, S., Dittrich, T. R., Haan, S. W., "Three-dimensional HYDRA simulations of National Ignition Facility targets", Physics of Plasmas 8, 2275 (2001).

[Dewald 2004] E. L. Dewald, K. M. Campbell, R. E. Turner, J. P. Holder, O. L. Landen, S. H. Glenzer, R. L. Kauffman, L. J. Suter, M. Landon, M. Rhodes, and D. Lee, "Dante soft x-ray power diagnostic for National Ignition Facility", Rev. Sci. Instrum. 75, 3759 (2004).

[Anderson 2004] R. W. Anderson, N. S. Elliott and R. B. Pember, "An arbitrary Lagrangian-Eulerian method with adaptive mesh refinement for the solution of the Euler equations", Journal of Computational Physics 199 (2), 20 September 2004, Pages 598-617.

[DYNA3D] Energy \& Technology Review, September-October, 1993, pp. 1-5 
[Froula 2009] D. H. Froula, et al., "Full-aperture backscatter measurements on the National Ignition Facility", Rev. Sci. Instrum. 75, 4168 (2004). J. Moody, et al., "Optical backscatter measurements to study laser plasma interactions on NIF", Bull. Am. Phys. Soc. 54, 142 (2009).

[Autodesk]

http://usa.autodesk.com/adsk/servlet/pc/index?siteID=123112\&id=13773836

[DRAT] http://dhmunro.github.com/yorick-doc/

[Zhuk 1994] A. Z. Zhuk, G. I. Kanel and A. A. Lash, Journal De Physique IV Colloque C8, supplement au Journal de Physique III (4), C8-403 (Sept. 1994)

[Fritz 1978] I. J. Fritz, J. Appl. Phys. 49(8) (August 1978).

[Harte 1996] J. A. Harte, "Lasnex - a 2-D Physics Code for Modeling ICF", ICF Quarterly Report 6 (4), UCRL-LR-105821-96-4 (1996).

[Zimmerman 1975] G. B. Zimmerman and W. L. Kruer, comments in Plasma Physics and Controlled Fusion 2, 51 (1975). 